\title{
Over-1000 nm near-infrared fluorescent biodegradable polymer nanoparticles for deep tissue in vivo imaging in the second biological window
}

Polymer Journal (2017) 49, 799-803; doi:10.1038/pj.2017.59; published online 27 September 2017

\section{INTRODUCTION}

Near-infrared (NIR) fluorescence bioimaging has received considerable attention in biomedical research owing to its attractive features, such as excellent tissue penetration and low phototoxicity. ${ }^{1,2}$ Most of the currently used fluorescent probes have several drawbacks, such as low body penetration and high phototoxicity, because they are excited by ultraviolet (UV; $<400 \mathrm{~nm}$ ) or short wavelength visible (VIS; 400-700 nm) light. As an alternative, NIR light solves these problems and provides a new opportunity to observe deep parts of the body with low phototoxicity. Because of this, the NIR wavelength range $(700-1800 \mathrm{~nm})$ is called the 'biological window' and is widely used for in vivo fluorescence imaging. ${ }^{3}$ In particular, the over-1000 nm (OTN-) NIR region is of wide interest as a novel wavelength region for use in fluorescence imaging. The OTN-NIR region consists of the 'NIR-II' (the second biological window: $1000-1350 \mathrm{~nm}$ ) and the 'NIR-III' (the third biological window: 1500-1800 nm) regions, and NIR light in these regions can penetrate the body deeper than the currently used NIR-I (first biological window: $700-900 \mathrm{~nm}$ ) region. $^{3-5}$ Various nanomaterials, such as quantum dots, ${ }^{6}$ single-wall carbon nanotubes ${ }^{7}$ and rareearth-doped ceramic nanoparticles, ${ }^{8-11}$ are well known to demonstrate OTN-NIR emission; many researchers, including our group, have reported successful in vivo fluorescence imaging using these nanomaterials. However, synthesis of these nanoparticles requires high levels of chemical experimental skill and is time consuming. In addition, these OTN-NIR phosphors comprise metal or inorganic nanomaterials, and thus are difficult to use in human clinical applications.
To address this, several researchers have recently attempted to use low-molecularweight OTN-NIR fluorescent dyes for in vivo imaging applications. ${ }^{12-14}$ Because low-molecular-weight compounds can be cleared from the blood by a renal clearance mechanism, low-molecular-weight dyes are attractive candidates for human clinical applications. Several kinds of laser dyes, such as IR-26, IR-1048 and IR-1061, are known to show OTN-NIR emission. However, because these kinds of OTN-NIR dyes possess cyanine or thiopyrilium structures, these dyes are poorly soluble in water, making it difficult to obtain stable OTN-NIR emission in an aqueous environment. Although IR-1061 can be soluble in water at low concentrations, it is difficult to disperse and circulate in blood at high concentrations.

To solve this problem, encapsulation of OTN-NIR dyes in biocompatible polymer nanoparticles for use in in vivo imaging has been reported. ${ }^{12,13}$ In these studies, dyes are loaded into the hydrophobic layer of polymer nanoparticles, thus allowing OTN-NIR emission in an aqueous environment. However, synthesis of these materials involves the use of several different kinds of polymer materials, a stepwise mixing process and/or formation of a layer-by-layer structure. ${ }^{12,13}$ Therefore, sample preparation requires a complicated procedure and a long preparation time. Furthermore, successful blood circulation of nanoparticles requires an adequate particle size. It is well known that nanoparticles ranging from 10 to $100 \mathrm{~nm}$ can circulate in the blood with a long half-life. ${ }^{15}$ In contrast, small size nanoparticles $(<10 \mathrm{~nm})$ are rapidly cleared by the kidneys, whereas large size nanoparticles $(>100 \mathrm{~nm})$ are trapped by the reticuloendothelial system. Additionally, a long blood half-life for the nanoparticle increase its accumulation at the tumor. This phenomenon is well known as the enhanced permeability and retention effect, and numerous anticancer drug delivery studies have been performed based on this phenomenon. ${ }^{16}$ Therefore, control of nanoparticle size is required for successful in vivo imaging. For these reasons, the development of a size-controllable OTN-NIR dye-loaded biocompatible polymer nanoparticle with an easy preparation method is required. Additionally, to address the safety problem after imaging experiments and diagnoses, a biodegradable polymer is also preferred for use as the polymer material. ${ }^{16,17}$ A biodegradable polymer can be degraded by hydrolytic cleavage, and thus can be easily cleared by the renal clearance mechanism.

In this study, we have developed OTN-NIR dye-loaded polymer micelles based on the OTN-NIR dye IR-1061 and the biocompatible and amphiphilic block copolymer, poly (ethylene glycol) (PEG)-block-poly( $\varepsilon$-caprolactone) (PCL) (PEG- $b$-PCL), for use as an OTN-NIR in vivo imaging fluorescence probe (Figure 1). PEG- $b$-PCL possesses both a hydrophilic PEG group and a hydrophobic PCL group, and thus can easily form a stable polymer micelle in an aqueous environment. The PEG- $b$-PCL micelle possesses a hydrophobic core and it can be used as a nanocarrier for hydrophobic compounds, such as anticancer drugs or fluorescent dyes. ${ }^{17}$ Because of the poor water solubility of IR-1061, it is effectively encapsulated into the hydrophobic core of the PEG- $b$-PCL micelles via a simple 'one-pot' procedure. Additionally, both the IR-1061 dye and PEG-b-PCL are commercially available reagents. Therefore, the IR-1061-loaded 


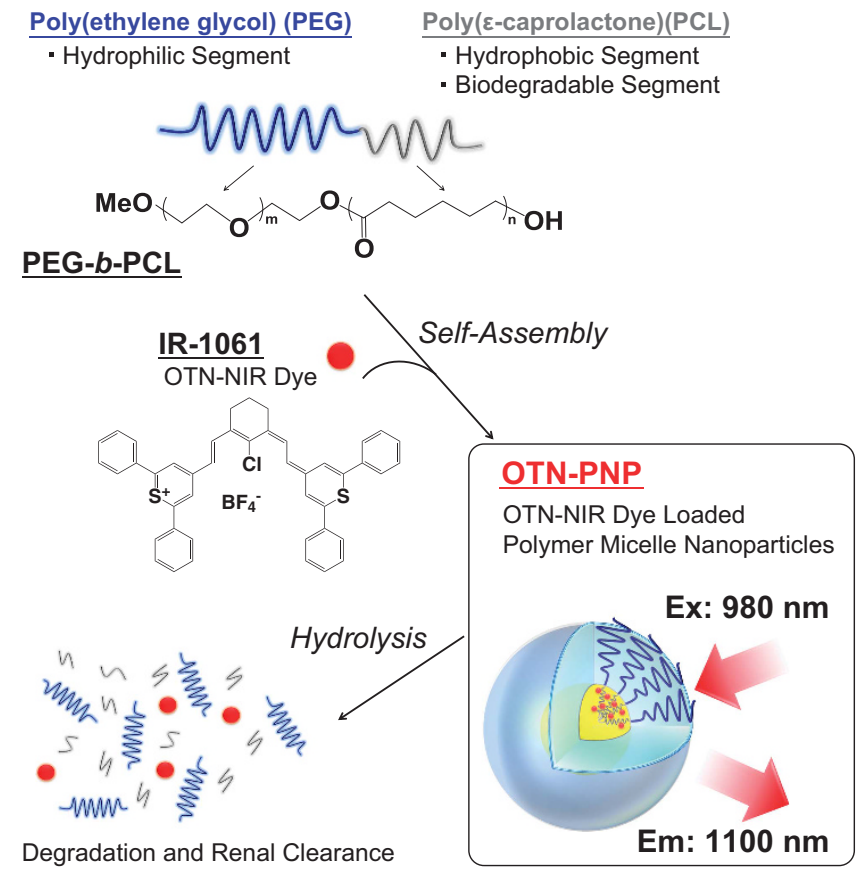

Figure 1 Schematic illustration of the design of OTN-PNPs.

PEG- $b$-PCL micelles (OTN-PNPs) can be easily prepared without special chemical experimental skills. Furthermore, the particle size of PEG- $b$-PCL micelles can be fine-tuned by changing the chain length of the polymers, the solvent amount and/or the polymer/ solvent ratio. ${ }^{18}$ Thus, the particle size of the OTN-PNPs can be easily controlled by the preparation conditions. In addition, because the PCL segment is biodegradable, the OTN-PNPs are expected to also be biodegradable. The preparation and the evaluation of OTN-PNPs as an OTN-NIR in vivo imaging fluorescence probe is discussed in detail below.

\section{MATERIALS AND METHODS \\ Materials}

PEG- $b$-PCL $\left(M_{\mathrm{n}}=5000 / 5000\right)$ was purchased from Polymer Source (Montreal, QC, Canada). IR-1061 was purchased from Sigma-Aldrich (St Louis, MO, USA). Acetonitrile $(\mathrm{ACN})$, sodium hydroxide $(\mathrm{NaOH})$ and penicillin-streptomycin were purchased from Wako Pure Chemical Industries (Osaka, Japan). WST reagent (Cell Counting Kit-8) was purchased from Dojindo Laboratories (Kumamoto, Japan). Minimum essential medium was purchased from Thermo Scientific (Waltham, MA, USA). Fetal bovine serum was purchased from Biowest (Nuaille, France). All reagents were used without further purification.

\section{Preparation of OTN-PNPs}

PEG- $b$-PCL solution (0.4 mm; $1 \mathrm{ml})$ in $\mathrm{ACN}$ and IR-1061 dye solution (130 mm; $100-350 \mu \mathrm{l}$ ) in ACN were mixed in a vial, followed by a rapid addition of distilled water. The final volume of $\mathrm{ACN} /$ water mixture samples were $3.0 \mathrm{ml}$. ACN was allowed to evaporate from the aqueous OTN-PNP solution during stirring at room temperature for $9 \mathrm{~h}$. The obtained OTN-PNPs were purified by centrifuge filter purification (MWCO $10000 \mathrm{Da}, 20000 \mathrm{~g}, 15 \mathrm{~min}, 3$ times) and then dispersed in distilled water.

\section{Characterization of OTN-PNPs}

The absorption spectra of the OTN-PNP samples were measured using a UV/VIS/ NIR spectrophotometer (U-4000, Hitachi, Japan). The hydrodynamic diameter of the OTN-PNP samples were determined by dynamic light scattering using LB-250 (Horiba, Kyoto, Japan). The emission spectra of the OTN-PNP samples were measured using a spectrometer (NIR-256-1.7; Avantes, Apeldoorn, Netherlands) equipped with a fiber-coupled laser diode (SP-976-5-1015-7; Laser Components, Olching, Germany) as the $980 \mathrm{~nm}$ excitation source.

\section{Hydrolytic degradation of OTN-PNPs}

The accelerated hydrolytic degradation of OTN-PNPs was performed under alkaline conditions. The OTN-PNPs were dispersed in $\mathrm{NaOH}$ solution $(0-1 \mathrm{M})$ and vigorously stirred for 3 days. The degradation of
OTN-PNPs was evaluated by dynamic light scattering measurement.

\section{Cell cytotoxicity}

HeLa cells were cultured in minimum essential medium containing $10 \%$ fetal bovine serum and $1 \%$ penicillin-streptomycin at $37^{\circ} \mathrm{C}$ in a humidified atmosphere containing $5 \% \mathrm{CO}_{2}$. The cell cytotoxicity of OTN-PNPs was assessed in HeLa cells by a standard WST proliferation assay. Briefly, cells were seeded in 96-well microtiter plates with $1 \times 10^{4}$ cells per well and allowed to adhere for $24 \mathrm{~h}$ prior assay. The cells were exposed to various concentration $\left(0.01-100 \mu \mathrm{g} \mathrm{ml}^{-1}\right.$ on IR-1061 dye) of the free IR-1061 dye or the OTN-PNP for $24 \mathrm{~h}$ at $37^{\circ} \mathrm{C}$, followed by washing with PBS, after which the cell viability was evaluated using the WST assay. Absorption was measured at $450 \mathrm{~nm}$ in a microplate reader (Multiskan FC; Thermo Scientific).

\section{OTN-NIR fluorescence in vivo imaging}

All animal care and experiments were conducted according to the guidelines on care and use of laboratory animals at the Tokyo University of Science. Male 3-week-old ICR mice were purchased from Sankyo Labo Service (Tokyo, Japan). Before in vivo imaging experiments, to lower the levels of body phosphorescent alfalfa, the mice were fed the AIN-76A diet (Research Diets, New Brunswick, NJ, USA) for 20 days. After this, the mice were anesthetized, and their hair was removed to avoid light scattering. Following this, $200 \mu \mathrm{l}$ of the OTN-PNP dispersion in physiological saline $\left(80 \mu \mathrm{g} \mathrm{ml}^{-1}\right.$ of dye) was injected into the tail vein. The OTN-NIR fluorescence images were observed using an OTN-NIR fluorescence in vivo imaging system (NIS-OPT, Shimadzu, Japan).

\section{RESULTS AND DISCUSSION}

Because of the OTN-NIR dye, IR-1061 has a strong absorption around the $1000 \mathrm{~nm}$ wavelength region; hence, it can be excited using a $980 \mathrm{~nm}$ laser excitation source. As shown in Supplementary Figure S1, IR-1061 has a strong OTN-NIR emission at $1110 \mathrm{~nm}$, which is in the NIR-II wavelength region. Because the penetrability of the NIR-II region is the highest in the OTN-NIR wavelength region, ${ }^{2,4}$ the IR-1061 dye has ideal fluorescence wavelengths for use as an OTN-NIR fluorescent probe. The OTN-PNPs were prepared by simple mixing of PEG- $b$-PCL and IR-1061 dye in a water/ACN mixture solution. Supplementary Figure S2 shows the emission spectra of OTN-PNPs with different nominal concentrations of OTN-NIR dye. As a result of this analysis, the maximum 


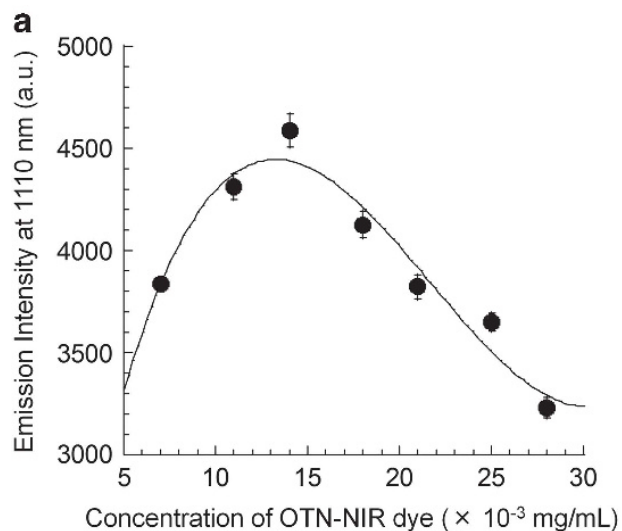

b
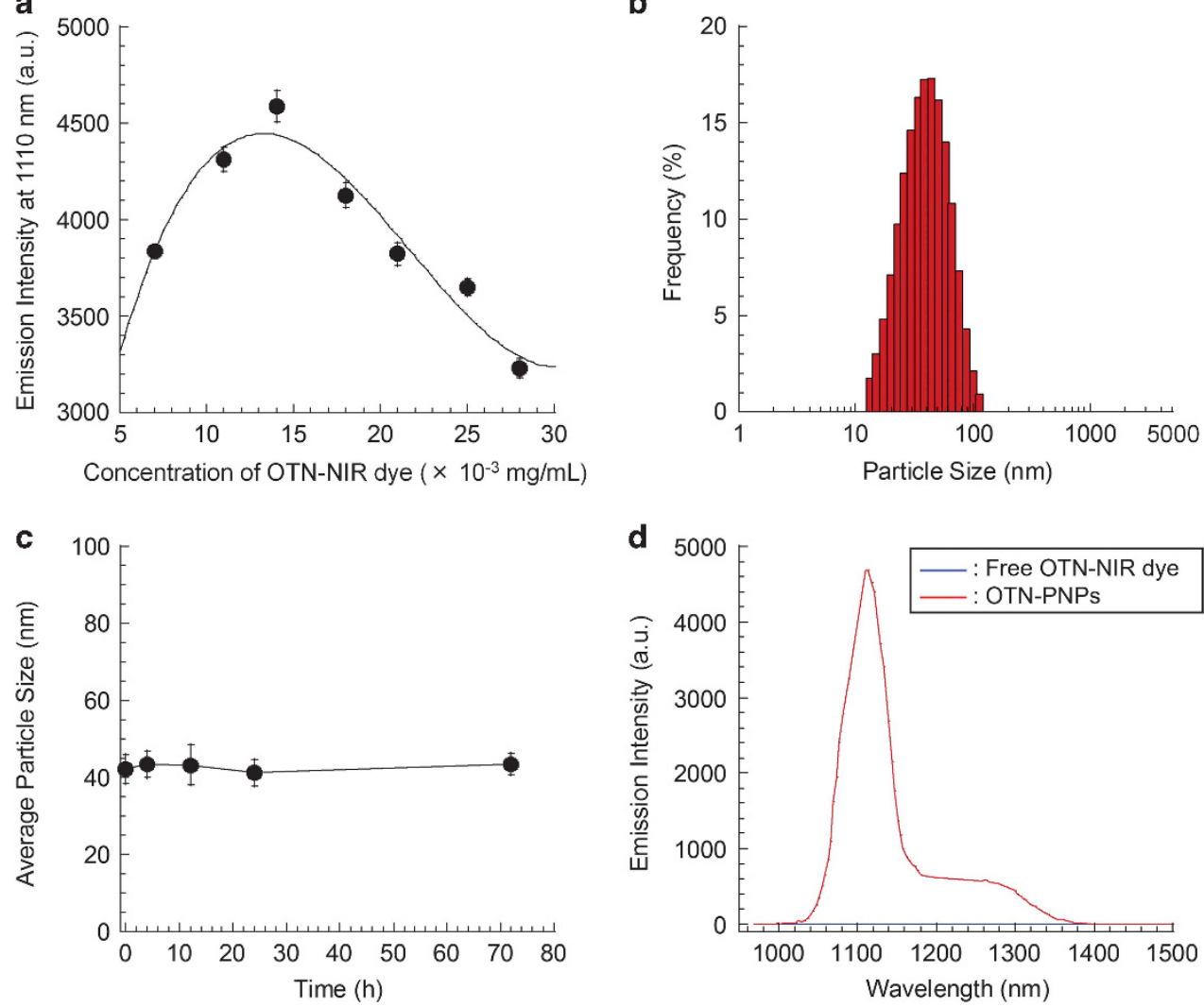

Figure 2 Characterization of OTN-PNPs. (a) Emission intensity of OTN-PNP at $1110 \mathrm{~nm}$ at different dye concentrations. The emission measurements were obtained using water as the dispersion medium. The excitation wavelength was $980 \mathrm{~nm}$ with a laser power of $4.22 \mathrm{~W}$. (b) Particle size distribution of OTN-PNPs in physiological saline measured by dynamic light scattering (DLS). (Measurement sample: $0.4 \%$ dye-loaded OTN-PNP.) (c) Time dependence of the average particle size of OTN-PNPs in physiological saline measured by DLS. (Measurement sample: $0.4 \%$ dye-loaded OTN-PNP.) (d) Emission spectra of OTN-NIR dye alone and OTN-PNPs in physiological saline. (Measurement sample: $0.4 \%$ dye-loaded OTN-PNP). The emission measurements were obtained using water as the dispersion medium. The excitation wavelength was $980 \mathrm{~nm}$ with a laser power of $4.22 \mathrm{~W}$.

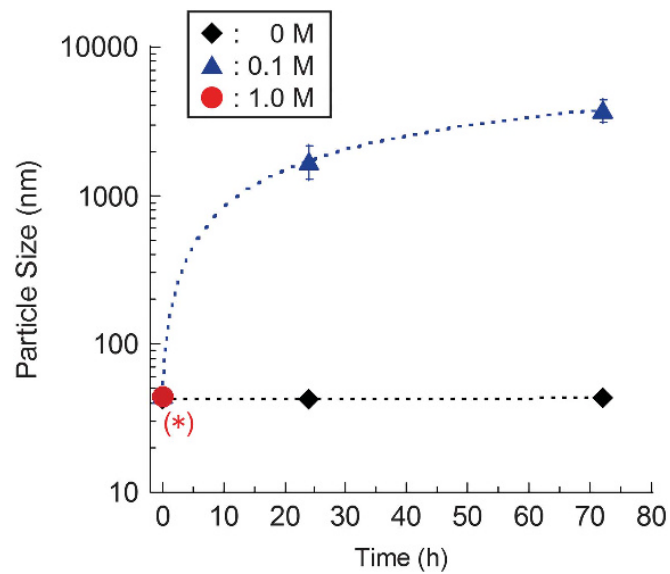

Figure 3 Hydrolysis of OTN-PNP at different $\mathrm{NaOH}$ concentrations (measurement sample: $0.4 \%$ dye-loaded OTN-PNP).

emission intensities of the OTN-PNPs at $1110 \mathrm{~nm}$ were found to be dependent on the dye concentration. Figure $2 \mathrm{a}$ shows the emission intensity changes of OTN-PNPs at $1110 \mathrm{~nm}$ at different dye concentrations. From these results, at dye concentrations $\leqslant 14 \times 10^{-3} \mathrm{mg} \mathrm{ml}^{-1}$, OTN-PNPs displayed a dye concentration-dependent increase in emission intensity. In contrast, at dye concentrations $>14 \times 10^{-3} \mathrm{mg} \mathrm{ml}^{-1}$, the emission intensity of the OTN-PNPs decreased with increasing dye concentrations. This is probably due to the high concentration of dye in the core of the OTN-PNPs causing fluorescence quenching. Therefore, at a dye concentration of $14 \times 10^{-3} \mathrm{mg} \mathrm{ml}^{-1}$, OTN-PNPs displayed the strongest OTNNIR emission intensity. In addition, the total dye loading of the OTN-PNPs obtained (dye concentration $=14 \times 10^{-3} \mathrm{mg} \mathrm{ml}^{-1}$ ) was $\sim 0.4 \%$ (expressed as mass of incorporated dye per mass of dye/PEG- $b$-PCL mixtures). Furthermore, the effect of dye concentration on the particle size of OTNPNPs was also evaluated. Supplementary Figure S3 shows the average particle size of OTN-PNPs at different dye concentrations. From these data, the average particle size of the OTN-PNPs was almost constant (ca. $40 \mathrm{~nm}$ ), regardless of dye concentrations. Therefore, we focused on a dye loading level of $0.4 \%$ in the OTN-PNPs for further evaluation.

The stability of the OTN-PNPs under physiological conditions was also evaluated. Figure $2 \mathrm{~b}$ shows the particle size distribution of OTN-PNPs in physiological saline. The average particle size of OTN-PNPs was $45 \pm 20 \mathrm{~nm}$, and it was almost the same size 


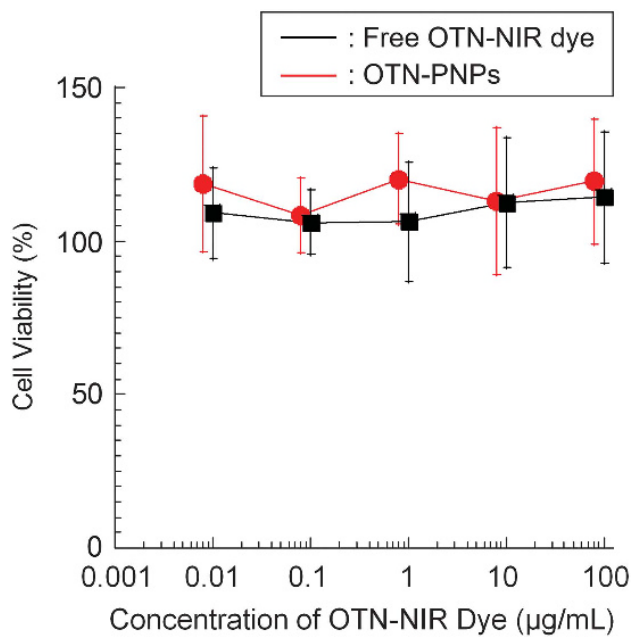

Figure 4 Cell cytotoxicity of OTN-PNPs against HeLa cells (measurement sample: $0.4 \%$ dye-loaded OTN-PNP).

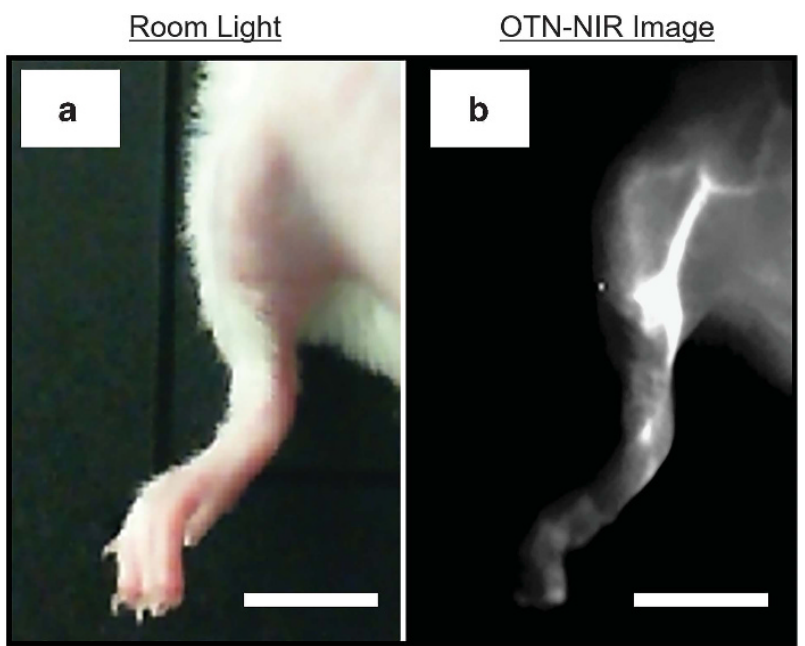

Figure 5 OTN-NIR fluorescence in vivo imaging of a live mouse. (a) Room light and (b) OTN-NIR image, respectively. (Emission measurement: excitation wavelength, $980 \mathrm{~nm}$; laser strength, $4.22 \mathrm{~W}$; scale bar: $10 \mathrm{~mm}$ ).

in pure water (Supplementary Figure S3). Furthermore, as shown in Figure 2c, the OTN-PNPs were also stable in physiological saline and no change in OTN-PNP particle size or size distribution was observed for up to 3 days. Moreover, the OTN-NIR emission of the OTN-PNPs in physiological saline was also recorded (Figure 2d). As mentioned above, the IR-1061 dye is poorly soluble in water, and thus it is difficult to measure OTN-NIR emission in an aqueous environment. As expected, the free dye did not show OTN-NIR emission at $980 \mathrm{~nm}$ excitation in physiological saline (Figure 2d). In contrast, the OTN-PNPs displayed a strong OTN-NIR emission in physiological saline (Figure 2d). These results indicate that the OTN-NIRs possess high dispersion and excellent emission stabilities.
Next, the biodegradability of the OTNPNPs was investigated using an accelerated hydrolytic degradation test. Because the PCL segment can be degraded by hydrolytic cleavage under acidic or alkaline conditions, ${ }^{19-21}$ PCL is well known as a biodegradable polymer. Generally, nanoparticles $(d<100 \mathrm{~nm})$ are taken up by cells via endocytosis pathway and it causes acidic hydrolysis. Therefore, OTN-PNPs are expected to show biodegradability in the body. However, acidic hydrolysis of nanoparticles under near-neutral conditions such as endosomal $\mathrm{pH}(6-6.5)$ is little difficult to observe in a short time, due to mild effect of acid. Therefore, in this study, as a proof of concept, commonly used accelerated hydrolytic degradation test under alkaline conditions was performed. ${ }^{19}$ The OTN-PNPs were dispersed in $\mathrm{NaOH}$ solutions $(0-1.0 \mathrm{M})$ and time-dependent changes in OTN-PNP particle size were evaluated (Figure 3). From this analysis, the average particle size of the OTN-PNPs did not change in normal water $(0 \mathrm{M} \mathrm{NaOH})$ for 3 days. However, in the case of the $\mathrm{NaOH}$ solutions, the particle size of the OTN-PNP samples changed markedly. In $0.1 \mathrm{M} \mathrm{NaOH}$ solution, an obvious time-dependent increase in the OTN-PNP particle size was observed. This is probably caused by cleavage of the ester groups in the PCL chains by alkaline hydrolysis leading to aggregation of the nanoparticles and/or the IR-1061 molecules. Furthermore, as denoted by an asterisk, in a $1.0 \mathrm{M} \mathrm{NaOH}$ solution, although the particle size of the OTN-PNPs could be measured just after dispersion in solution $(0 \mathrm{~h})$, it could not be measured $24 \mathrm{~h}$ later. This is also probably due to the fact that the OTN-PNPs were degraded immediately in the high alkali conditions. These results suggest that the ONT-PNPs are likely to be biodegradable.

For clinical use, a fluorescent probe with low toxicity is important. To begin to address this, the cell cytotoxicity of the OTN-PNPs were evaluated using a standard WST assay (Figure 4). As a result, the free IR-1061 dye did not show any obvious cell cytotoxicity. Additionally, OTN-PNPs also have little effect on cell viability even at high concentrations $\left(\leqslant 100 \mu \mathrm{g} \mathrm{ml}^{-1}\right)$. Therefore, we conclude that OTN-PNPs have minimal toxicity and are likely to have good biological safety. From these data, we anticipated that the OTN-PNPs could be successfully used as OTN-NIR imaging probes.

Finally, we examined blood vessel imaging of live mice using the OTN-PNPs. An important feature of the OTN-PNPs is their ability to obtain high-resolution imaging of blood vessels in live mice. For blood vessel imaging, OTN-PNPs were injected into male ICR mice via the tail vain and the OTN-NIR fluorescence image was obtained up to $1 \mathrm{~min}$ post injection. Figure 5 shows the room light image (a) and OTN-NIR fluorescence image (b) of the OTN-PNP-injected mice. From these data, Figure $5 \mathrm{~b}$ shows that the blood vessels of the right leg of a live mouse can be observed, without the observation window or dissection. This result also suggests that OTN-PNPs have a high potential for use in vivo fluorescence imaging of the deep parts of a mouse body.

\section{CONCLUSION}

In conclusion, using the OTN-NIR dye IR-1061-loaded polymer micelles, OTN-PNPs were successfully obtained using a simple one-pot preparation method. The prepared OTN-PNPs were well dispersed and 
had a strong OTN-NIR emission under physiological conditions. Furthermore, the OTN-PNPs were readily biodegradable; thus, it is anticipated that the renal clearance of the degraded material will occur after imaging experiments. Additionally, the OTN-PNPs showed minimal cell cytotoxicity over the range of doses that could be used in in vivo imaging. Moreover, in vivo imaging of a live mouse was successfully performed. The blood vessels of a live mouse could be clearly illuminated using the OTN-NIR emission of OTN-PNPs. Therefore, the OTN-PNPs obtained in this study are promising candidates for NIR imaging probes.

\section{CONFLICT OF INTEREST}

The authors declare no conflict of interest.

\section{ACKNOWLEDGEMENTS}

This research was supported by a Grant-in-Aid for Scientific Research on Innovative Areas (Resonance Bio), No. 15H05950, from the Ministry of Education, Culture, Sports, Science and Technology of Japan.

Masao Kamimura ${ }^{1,2}$, Shoko Takahiro ${ }^{1}$, Moe Yoshida ${ }^{1}$, Yusuke Hashimoto ${ }^{1}$, Rihito Fukushima ${ }^{1}$ and Kohei Soga ${ }^{1,2}$

${ }^{1}$ Department of Materials Science and Technology, Tokyo University of Science, Tokyo, Japan and ${ }^{2}$ Imaging Frontier Center (IFC), Research Institute for Science and Technology (RIST), Tokyo University of Science, Tokyo, Japan

E-mail: masaokamimura@rs.tus.ac.jp or mail@ksoga.com

1 Jaque, D., Richard, C., Viana, B., Soga, K., Liu, X \& Solé, J. G. Inorganic nanoparticles for optical bioimaging. Adv. Opt. Photonics 8, 1-103 (2016).
2 Hong, G., Antaris, A. L. \& Dai, H. Near-infrared fluorophores for biomedical imaging. Nat. Biomed. Eng. 1, 0010 (2017).

3 Smith, A. M., Mancini, M. C. \& Nie, S. Bioimaging: second window for in vivo imaging. Nat. Nanotechnol. 4, 710-711 (2009).

4 Hemmer, E., Benayas, A., Légaré, F. \& Vetrone, F. Exploiting the biological windows: current perspectives on fluorescent bioprobes emitting above $1000 \mathrm{~nm}$. Nanoscale Horiz. 1, 168-184 (2016).

5 Hemmer, E., Acosta-Mora, P., Mendez-Ramos, J. \& Fischer, S. Optical nanoprobes for biomedical applications: shining a light on upconverting and near-infrared emitting nanoparticles for imaging, thermal sensing, and photodynamic therapy. J. Mater. Chem. B 5, 4365-4392 (2017).

6 Nakane, Y., Tsukasaki, Y., Sakata, T., Yasuda, H. \& Jin, T. Aqueous synthesis of glutathione-coated $\mathrm{PbS}$ quantum dots with tunable emission for non-invasive fluorescence imaging in the second near-infrared biological window (1000-1400 nm). Chem. Commun. 49 7584-7586 (2013).

7 Welsher, K., Liu, Z., Sherlock, S. P., Robinson, J. T. Chen, Z., Daranciang, D. \& Dai, H. A route to brightly fluorescent carbon nanotubes for near-infrared imaging in mice. Nat. Nanotechnol. 4, 773-780 (2009).

8 Kamimura, M., Kanayama, N., Tokuzen, K., Soga, K. \& Nagasaki, Y. Near-infrared $(1550 \mathrm{~nm})$ in vivo bioimaging based on rare-earth doped ceramic nanophosphors modified with PEG-b-poly(4vinylbenzylphosphonate). Nanoscale 3, 3705-3713 (2011).

9 Naczynski, D. J., Tan, M. C., Zevon, M., Wall, B. Kohl, J., Kulesa, A., Chen, S., Roth, C. M., Riman, R. E. \& Moghe, P. V. Rare-earth-doped biological composites as in vivo shortwave infrared reporters. Nat. Commun. 4, 2199 (2013).

10 Kamimura, M., Saito, R., Hyodo, H., Tsuji, K., Umeda, I. O., Fujii, H. \& Soga, K. Over-1000 nm near-infrared fluorescence and SPECT/CT dual-modal in vivo imaging based on rare-earth doped ceramic nanophosphors. J. Photopolym. Sci. Technol. 29, 525-532 (2016).

11 Kamimura, M., Matsumoto, T., Suyari, S., Umezawa, M. \& Soga, K. Ratiometric near-infrared fluorescence nanothermometry in the OTN-NIR (NIR II/III) biological window based on rare-earth doped [small beta]-NaYF4 nanoparticles. J. Mater. Chem. B 5, 1917-1925 (2017).

12 Tao, Z., Hong, G., Shinji., C., Chen, C., Diao, S. Antaris, A. L., Zhang, B., Zou., Y. \& Dai, H. Biological imaging using nanoparticles of small organic molecules with fluorescence emission at wavelengths longer than 1000 nm. Angew. Chem. Int. Ed. 52, 13002-13006 (2013).

13 Dang, X., Gu, L., Qi, J., Correa, S., Zhang, G., Belcher, A. M. \& Hammond, P. T. Layer-by-layer assembled fluorescent probes in the second nearinfrared window for systemic delivery and detection of ovarian cancer. Proceedings of the National Academy of Sciences 113, 5179-5184 (2016).

14 Antaris, A. L., Chen, H., Cheng, K., Sun, Y., Hong, G. Qu, C., Diao, S., Deng, Z., Hu, X., Zhang, B., Zhang, X., Yaghi, O. K., Alamparambil, Z. R., Hong, X., Cheng, Z.

\& Dai, H. A small-molecule dye for NIR-II imaging. Nat. Mater. 15, 235-242 (2016).

15 Matsumura, Y. \& Maeda, H. A new concept for macromolecular therapeutics in cancer chemotherapy: mechanism of tumoritropic accumulation of proteins and the antitumor agent Smancs. Cancer Res. 46, 6387-6392 (1986).

16 Kataoka, K., Harada, A. \& Nagasaki, Y. Block copolymer micelles for drug delivery: design, characterization and biological significance. Adv. Drug Deliv. Rev. 47, 113-131 (2001).

17 Cho, H. \& Kwon, G. S. Polymeric micelles for neoadjuvant cancer therapy and tumor-primed optical imaging. ACS Nano 5, 8721-8729 (2011).

18 Vakil, R. \& Kwon, G. S. Poly(ethylene glycol)- $b-$ poly(e-caprolactone) and PEG-phospholipid form stable mixed micelles in aqueous media. Langmuir 22 9723-9729 (2006).

19 Asoh, T. -A., Nakajima, T., Matsuyama, T. \& Kikuchi, A. Surface-functionalized biodegradable nanoparticles consisting of amphiphilic graft polymers prepared by radical copolymerization of 2-methylene-1,3-dioxepane and macromonomers. Langmuir 31, 6879-6885 (2015).

20 Geng, Y. \& Discher, D. E. Hydrolytic degradation of poly(ethylene oxide)-block-polycaprolactone worm micelles. J. Am. Chem. Soc. 127, 12780-12781 (2005).

21 Sisson, A. L., Ekinci, D. \& Lendlein, A. The contemporary role of $\varepsilon$-caprolactone chemistry to create advanced polymer architectures. Polymer 54, 4333-4350 (2013).

\section{(i) (2)}

This work is licensed under a Creative Commons

Attribution-NonCommercial-ShareAlike $\quad \mathbf{4 . 0}$ International License. The images or other third party material in this article are included in the article's Creative Commons license, unless indicated otherwise in the credit line; if the material is not included under the Creative Commons license, users will need to obtain permission from the license holder to reproduce the material. To view a copy of this license, visit http://creativecommons.org/ licenses/by-nc-sa/4.0/

(C) The Author(s) 2017

Supplementary Information accompanies the paper on Polymer Journal website (http://www.nature.com/pj) 